\title{
Sterile Seroma Resulting from Multilevel XLIF Procedure as Possible Adverse Effect of Prophylactic Vancomycin Powder: A Case Report
}

\author{
jim A. Youssef ${ }^{1} \quad$ Douglas G. Orndorff ${ }^{1} \quad$ Morgan A. Scott $^{1} \quad$ Rachel E. Ebner $^{1} \quad$ Allison P. Knewitz ${ }^{1}$ \\ ${ }^{1}$ Spine Colorado, Durango, Colorado, United States \\ Address for correspondence Jim A. Youssef, MD, 1 Mercado Street, \\ Evid Based Spine Care J 2014;5:127-133. \\ Suite 202, Durango, CO 81301, United States \\ (e-mail: jyoussef@spinecolorado.com).
}

\begin{abstract}
Study Design Case report.

Objective The objective of this study was to present the unusual case of a 59-year-old woman with a reoccurring sterile postoperative seroma.

Methods A patient was observed postoperatively for any complications or adverse side effects resulting from an initial multilevel anterior/posterior lumbar fusion surgery where $2 \mathrm{~g}$ ( $1 \mathrm{~g}$ combined with the bone graft used for posterolateral fusion and $1 \mathrm{~g}$ placed in the soft tissues) of prophylactic vancomycin powder was placed within the soft tissues posteriorly before wound closure. The patient's progress was monitored through 6 months following the initial procedure. Six weeks postoperatively, the patient sustained a fall and had increased pain. Magnetic resonance imaging, computed tomography, and X-rays demonstrated a displaced sacral fracture, a large epidural fluid collection, and severe compression of the thecal sac at the lumbar operative sites (L3-5).

Results On the basis of the aforementioned imaging studies and the patient's progressive neurologic deficit, it was apparent at the 6-week follow-up that emergent surgical intervention was necessary. Drainage and examination of an epidural fluid collection along with treatment of a displaced sacral fracture (S1-S2) were performed. The patient had an uneventful postoperative course with resolution of her back pain and neurologic deficit; however, recurrence of the epidural fluid collection requiring serial aspirations confounded the patients' clinical presentation.

Conclusions With the recurrent nature of the seroma being unusual, the cause of the fluid collection and formation is undetermined. With lack of bone morphogenetic

Keywords

- vanomycin

- seroma

- lumbar fusion protein usage, and few confounding variables accountable, an acute allergic response to topical vancomycin powder is a possible etiology. Analysis with larger patient populations comparing postoperative adverse effects of prophylactic vancomycin powder is recommended.
\end{abstract}

\section{Introduction}

A sterile seroma may be the result of antibodies against collagen or a hyperresponder/allergic reaction. ${ }^{1}$ With seroma formation, severe compression can occur on the thecal sac causing spinal stenosis resulting in weakness and pain. Intraoperatively cell death and vascular permeability can occur along with an inflammatory reaction that can lead to fluid received

March 14, 2014

accepted

June 25,2014 (c) 2014 Georg Thieme Verlag KG Stuttgart · New York
DOI http://dx.doi.org/ 10.1055/s-0034-1386754. ISSN 1663-7976. 
accumulation. With studies linking usage of local vancomycin powder to lower rates of surgical site infection, ${ }^{2}$ its application has become more routine in complex cases since 2012. The local delivery of vancomycin powder provides high local concentration of the antibiotics with limited systemic toxicity making it a popular form of reducing infection rates. ${ }^{3}$ The efficacy of prophylactic vancomycin powder is still a topic of focus with studies on postoperative infection rates showing varied results and little concentration on possible adverse effects. $^{2-5}$ Its prevention or correlation to noninfectious surgical complications however has not yet been established.

\section{Case Report}

A 59-year-old female patient with a height of 5'6 and body mass index (BMI) of 27.1. The initial symptoms included lower back pain with bilateral leg pain, neurogenic claudication, as well as weakness in her right lower extremity (L3-L5 distribution). Previous X-rays and magnetic resonance imaging (MRI) showed degenerative scoliosis of the lumbar spine, grade 1 hypermobile anterolisthesis of L5 on S1, severe disc collapse at L5-S1, vacuum disc phenomenon at L2-L3, focal retrolisthesis at L2-L3, spondylosis from L2-S1, and severe spinal stenosis from L2-S1. Nonoperative efforts were exhausted primarily, followed by the recommendation for surgical intervention. The preoperative diagnosis was lumbar spondylosis at L1-S1, stenosis with neurogenic claudication, bilateral radicular features and neurologic deficit from $\mathrm{L} 2$ to

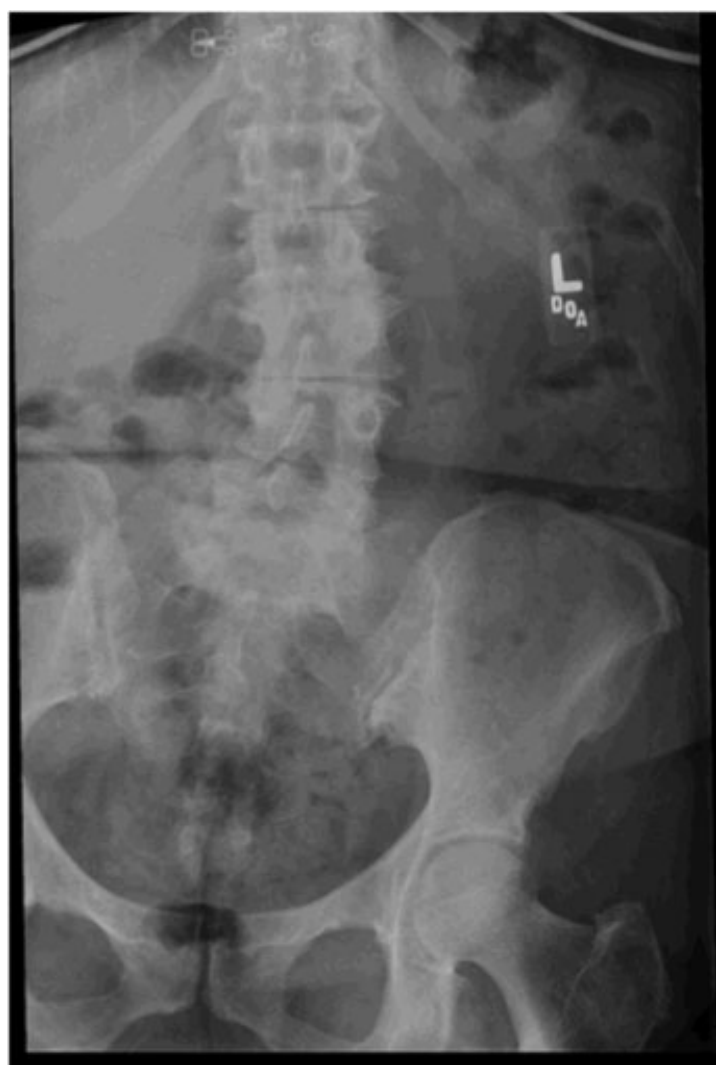

Fig. 1 Preoperative X-ray image of coronal plane.

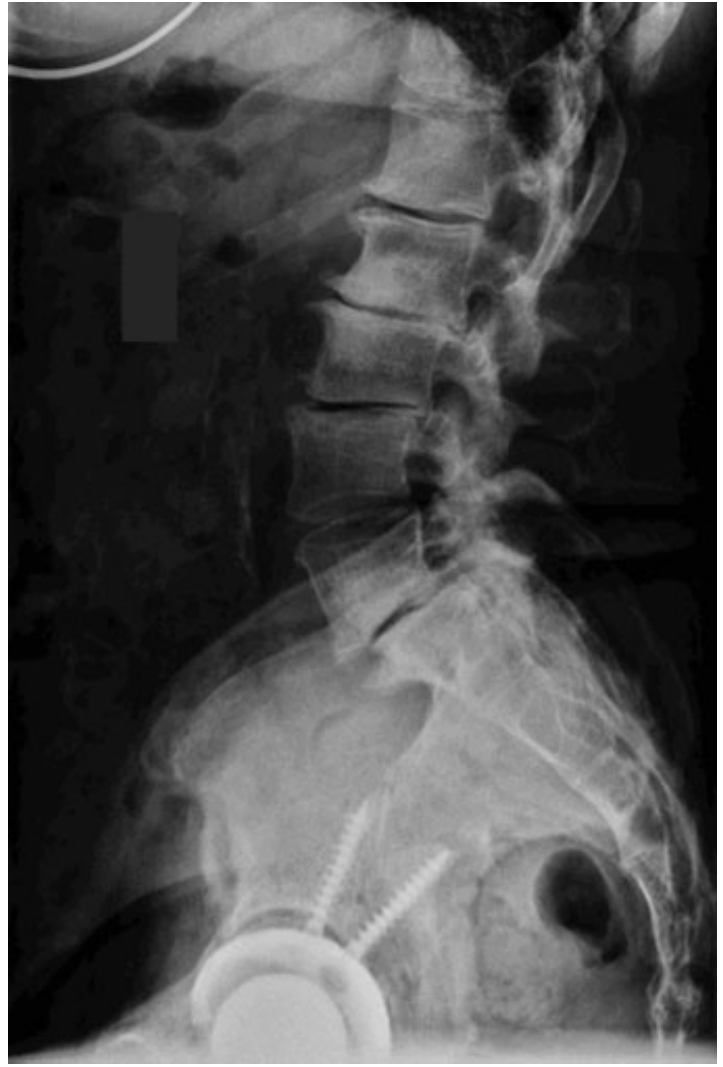

Fig. 2 Preoperative lateral X-ray image.

S1, degenerative scoliosis from L1 to S1, and type 1, grade 1 spondylolisthesis from L5 to S1. Preoperative X-ray and MRI images are shown in -Figs. 1 to 4 . The confounding variables considered include age, levels operated on, estimated blood loss, drain usage, and BMI shown in - Table 1.

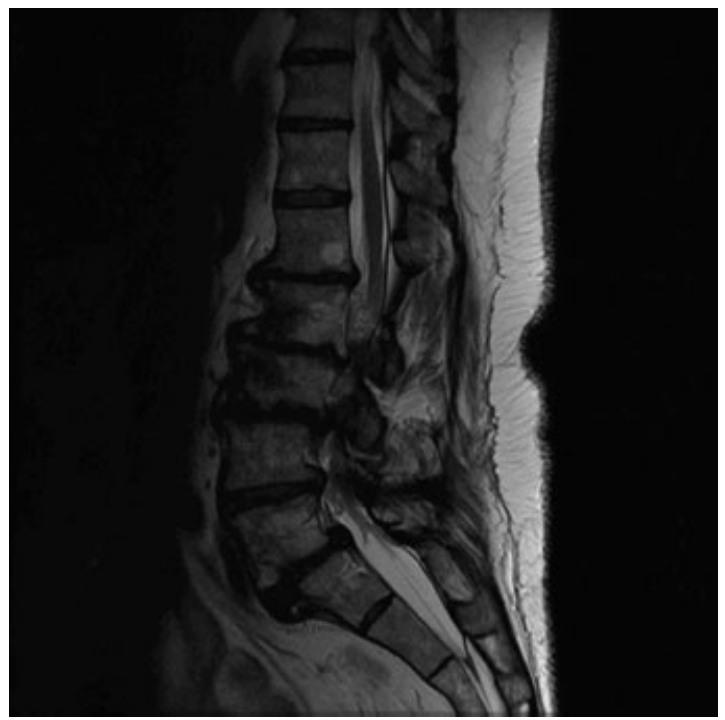

Fig. 3 Preoperative magnetic resonance imaging of sagittal plane. 


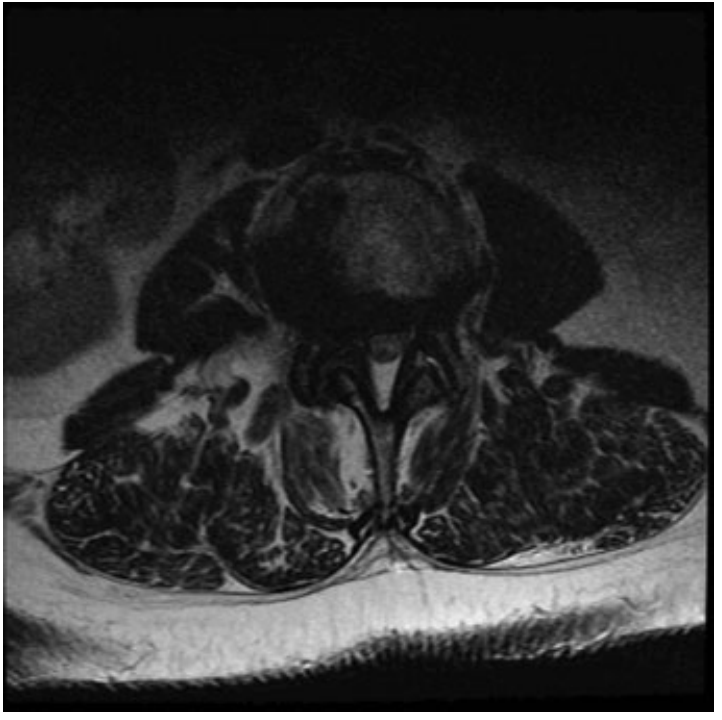

Fig. 4 Preoperative magnetic resonance imaging of transverse plane.

An Extreme Lateral Interbody Fusion (XLIF; NuVasive, San Diego, California, United States) procedure was performed at L1-L5 with application of PEEK implants (CoRoent, NuVasive) and packed with allograft bone (Osteocel Plus, NuVasive) at each level. This was followed by a same day decompressive laminectomy with bilateral complete facetectomies and foraminotomies from L2 to L5. Posterior spinal fusion was completed from L1 to S1 with posterior segmental pedicle screw instrumentation and autogenous bone graft placed from L2 to S1. A transforaminal interbody fusion was performed at L5-S1 with application of a ceramic interbody implant (Valeo TL Lumbar Interbody Fusion Device, Amedica Corporation, Salt Lake City, Utah, United States) that was also packed with allograft bone. Morselized autogenous local bone graft, obtained from the lamina and spinous processes, were combined with $1 \mathrm{~g}$ of vancomycin powder was evenly distributed along the posterolateral gutters from L1 to S1 serving as the posterior arthrodesis. An additional gram of vancomycin was placed along the soft tissues and paraspinous musculature, and attention was then drawn to wound closure.

Table 1 Confounding variables taken into consideration, with values representing initial surgery

\begin{tabular}{|l|l|}
\hline Age $(\mathrm{y})$ & 59 \\
\hline BMI & 27.1 \\
\hline Smoker & Previous smoker \\
\hline rhBMP-2 usage, $\mathrm{mg}$ & 0 \\
\hline Estimated blood loss, $\mathrm{mL}$ & 800 \\
\hline Vancomycin powder, $\mathrm{g}$ & 1 \\
\hline Drain usage & $\begin{array}{l}2 \text { Closed Wound Suction Kits } \\
\text { (C. R. Bard, Covington, Georgia, } \\
\text { United States) }\end{array}$ \\
\hline Levels operated on & 5 (L1-S1) \\
\hline
\end{tabular}

Abbreviations: BMI, body mass index; rhBMP-2, recombinant human bone morphogenetic protein.

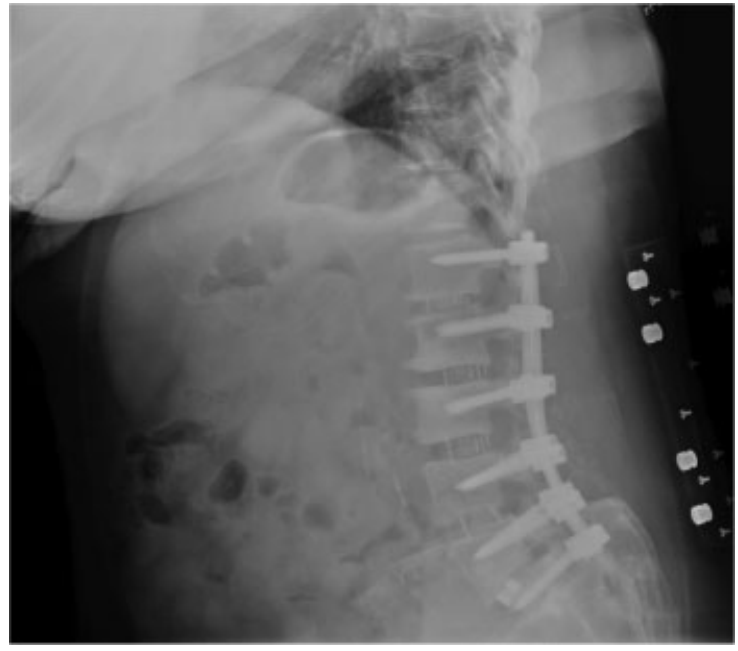

Fig. 5 A 2.5-week lateral X-ray image.

Before closure, two Hemovac drains were brought through a separate incision in an attempt to reduce hematoma formation. There were no complications intraoperatively.

At her 2-week routine postoperative visit, the patient presented with improved leg pain, expected postoperative back pain, and some approach-related hip flexion pain. X-rays were taken at this time (-Fig. 5). At 6 weeks postoperatively, the patient presented with a 2-day history of sustaining a fall after missing a chair while attempting to sit down, progressive and increasing back pain, as well as left-sided leg weakness with associated foot drop, difficulty standing, and ambulating. X-rays were repeated at this visit (-Figs. 6 and 7), and an emergent MRI was performed based on the clinical findings and neurologic deficit (- Figs. 8 and 9). An L2-S1 epidural fluid collection was identified on the MRI, causing severe neural compression. In addition, a displaced sacral fracture from S1 to S2 just below the disc space shown in the sagittal plane was diagnosed (-Fig. 8). Emergent surgery was recommended and performed to address the compressive fluid collection and the fracture.

The differential diagnosis for this patient's second surgery included possible cerebrospinal fluid leak (CSF) leak, infection, or seroma/hematoma, as well as the displaced S1-S2 sacral fracture. The surgery that followed included: incision and drainage of both the superficial and deep liquefied seroma, posterior instrumentation from L4 to the ilium, and bilateral iliac screws and rod connectors (-Figs. 10-13). Samples of the extracted fluid were sent to a laboratory for culture and sensitivity and $\beta-2$ transferrin analysis, in which there were no findings of CSF or infection. Aerobic and anaerobic gram cultures showed no growth after 7 days, and no organisms were observed in the fluid. A $\beta-2$ transferrin test was performed showing no detection of CSF fluid. There were no complications intraoperatively.

Four weeks postrevision surgery, a reaccumulation of serosanguineous fluid requiring aspiration was observed. This was observed clinically without any other signs or symptoms of wound infection or progressive neurologic deficit. In fact, the patient continued to improve neurologically, ambulated 


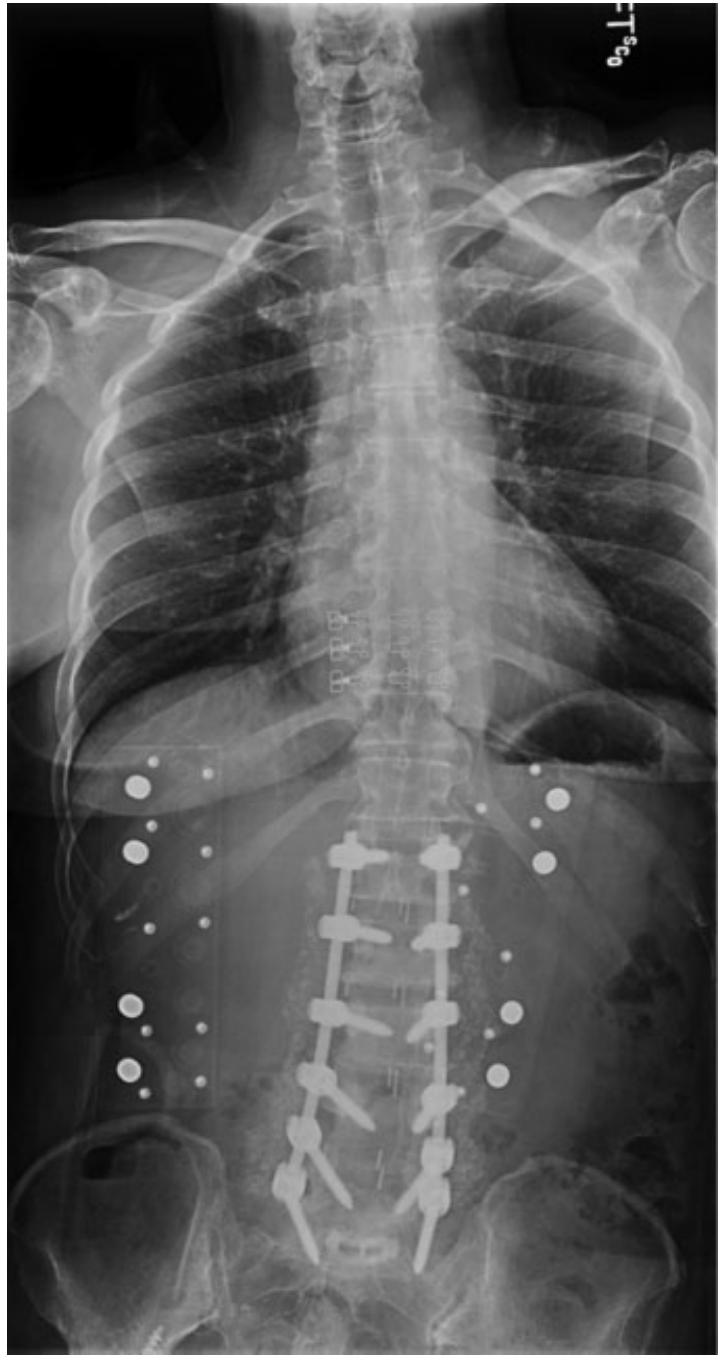

Fig. 6 A 6-week scoliosis anteroposterior image.

without back or leg pain, and remained afebrile. The patient had routine laboratories performed with normal complete blood count values and erythrocyte sedimentation rate of 25 $\mathrm{mm} / \mathrm{h}$. The results were all reasonable levels, and presented no unusual findings. This accumulation of fluid required aspiration a total of eight additional times within 3 months of the revision surgery. Following each of multiple aspirations, the fluid was cultured and sent to microbiology yielding the same results showing no infection. Initially, a total of $60 \mathrm{~mL}$ of fluid was aspirated. This decreased during each aspiration to a final amount of $10 \mathrm{~mL}$ at the last aspiration. The spacing between the times of drainage was initially every week, slowly increasing in time to drainage every 2 to 3 weeks.

\section{Discussion}

Despite the sterility of the seroma formed, its persistency can cause significant complications and pain for the patient due to pressure being exerted onto the thecal sac. It is thought that seroma formation may be due to a local inflammatory response, or the presence of foreign bodies; however, the exact causation of its formation is unknown. ${ }^{3}$ The recurrent

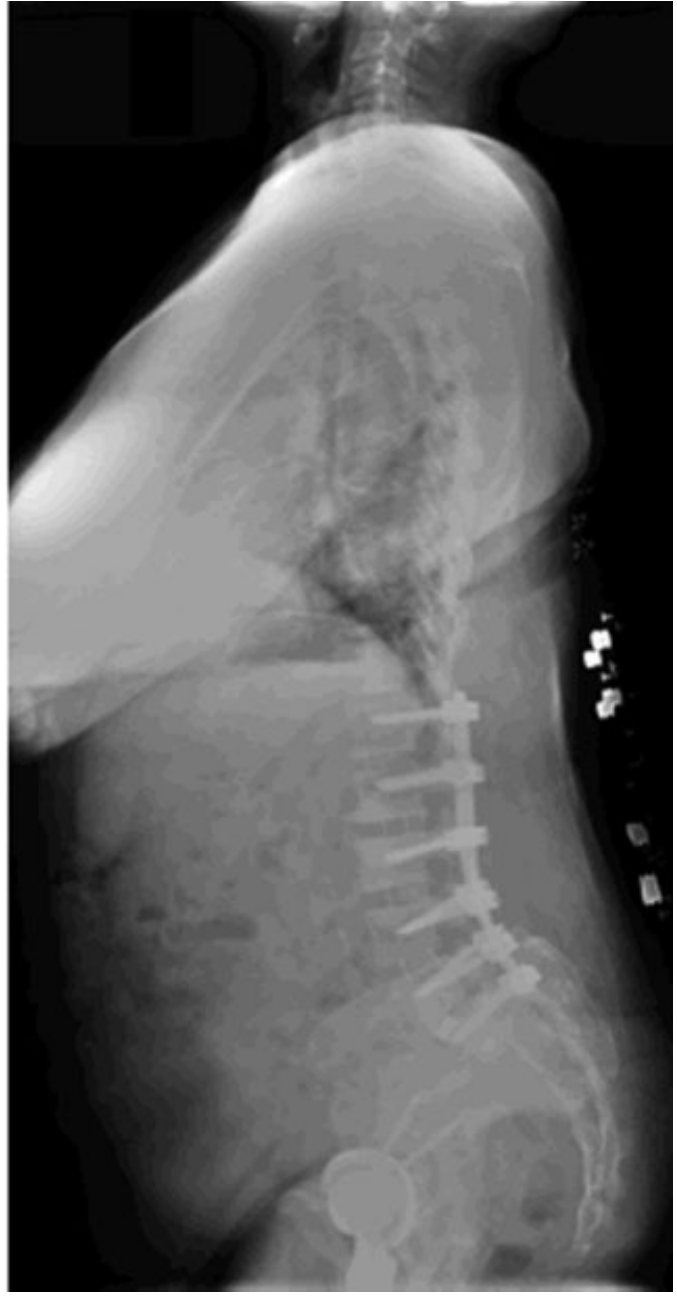

Fig. 7 A 6-week lateral X-ray image.

nature of the sterile seroma formed is an unusual case and has become a complication requiring consistent management.

In the results of an analysis of more than 14,000 patients, it was shown that risk factors of epidural hematoma formation

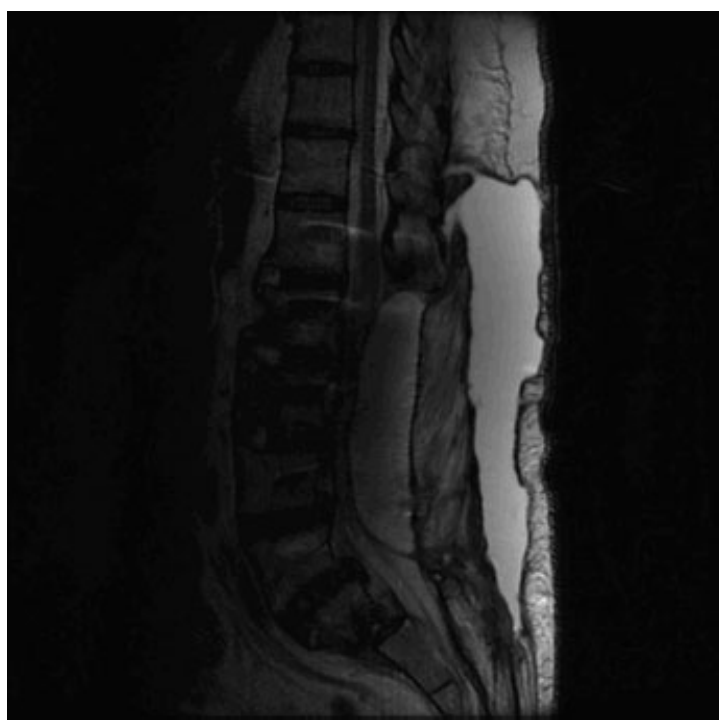

Fig. 8 A 6-week magnetic resonance imaging of sagittal plane. 


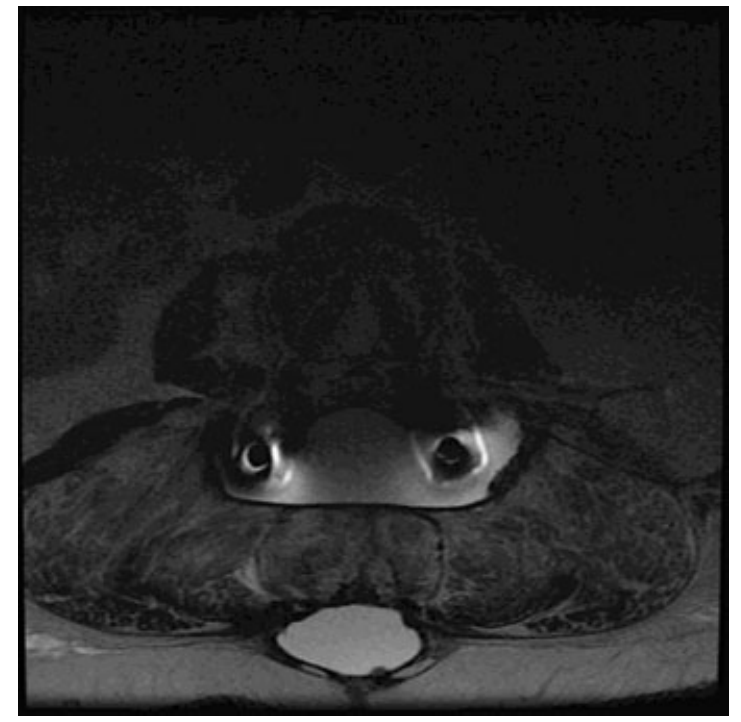

Fig. 9 A 6-week magnetic resonance imaging of transverse plane.

include anemia ( $<10 \mathrm{~g} / \mathrm{dL}$ ), an age of older than 60 , excessive blood loss (> $1 \mathrm{~L}$ ), or more than five operative levels. ${ }^{6}$ Research indicates no direct correlation between hematoma formation and lack of subfascial drains, tobacco usage, or usage of well-controlled anticoagulation. ${ }^{6}$ For this patient, the only possible confounding risk factor was the number of levels operated on. The current usage of closed-suction drains is a common technique used to remove excess fluid from around the incision site; however, the effectiveness of the closed-suction drain in high-risk surgeries is undetermined and most studies suggest no correlation to lower seroma or hematoma formation. 6,7

In addition, there have been multiple studies correlating the usage of recombinant human bone morphogenetic

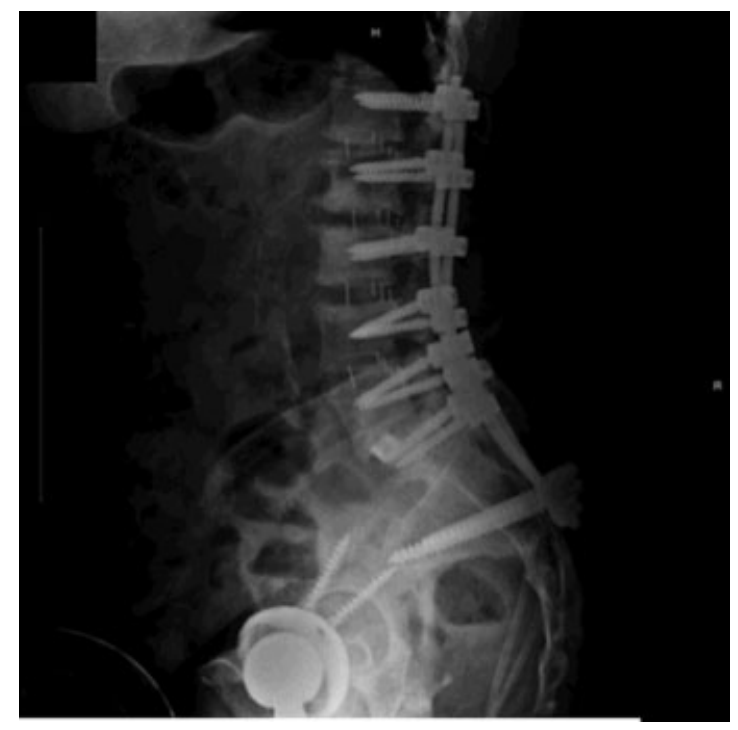

Fig. 10 A 2.5-week lateral X-ray image post emergent surgery.

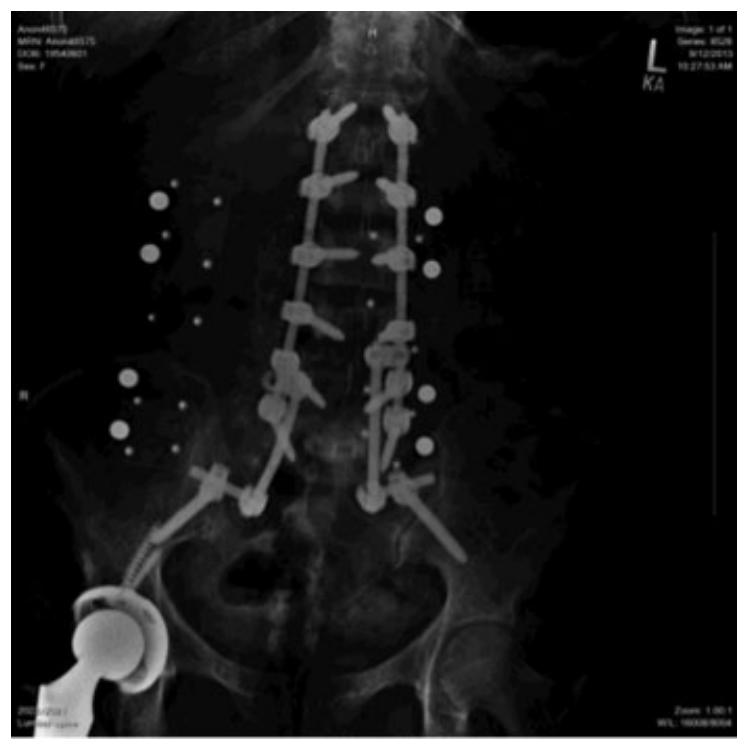

Fig. 11 A 2.5-week X-ray image post emergent surgery of coronal plane.

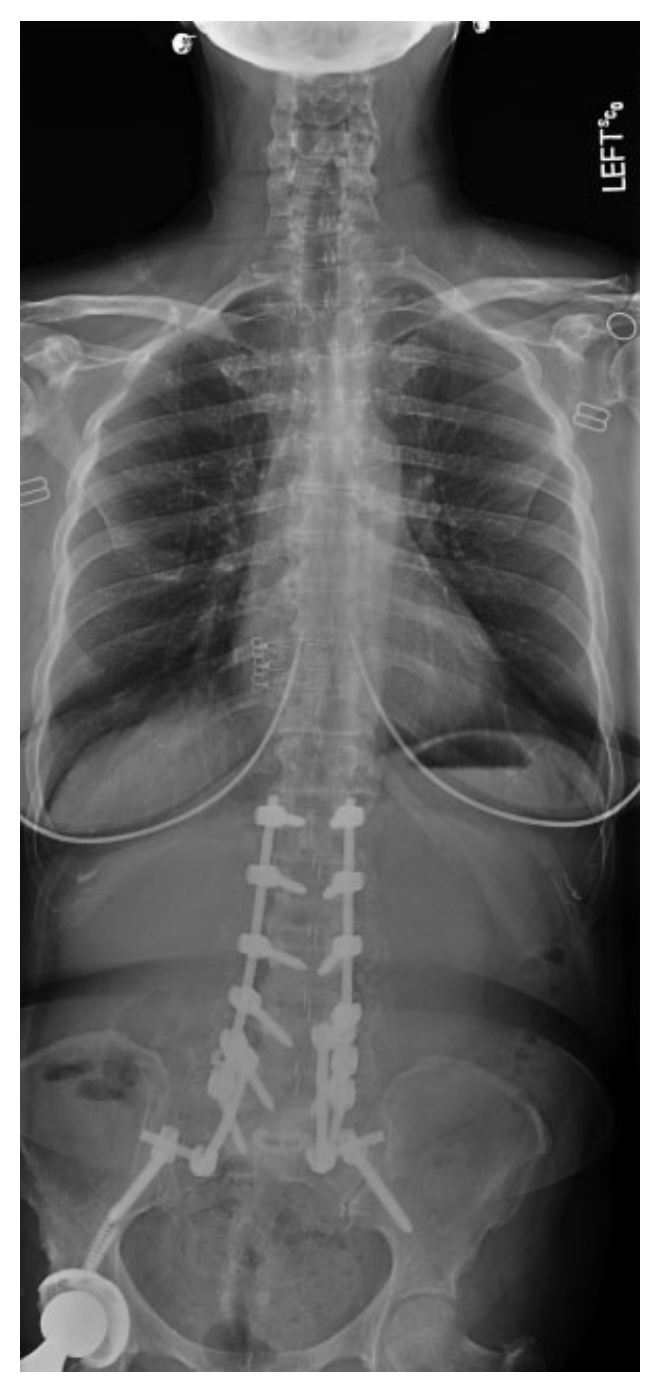

Fig. 12 A 6-month scoliosis anteroposterior image. 


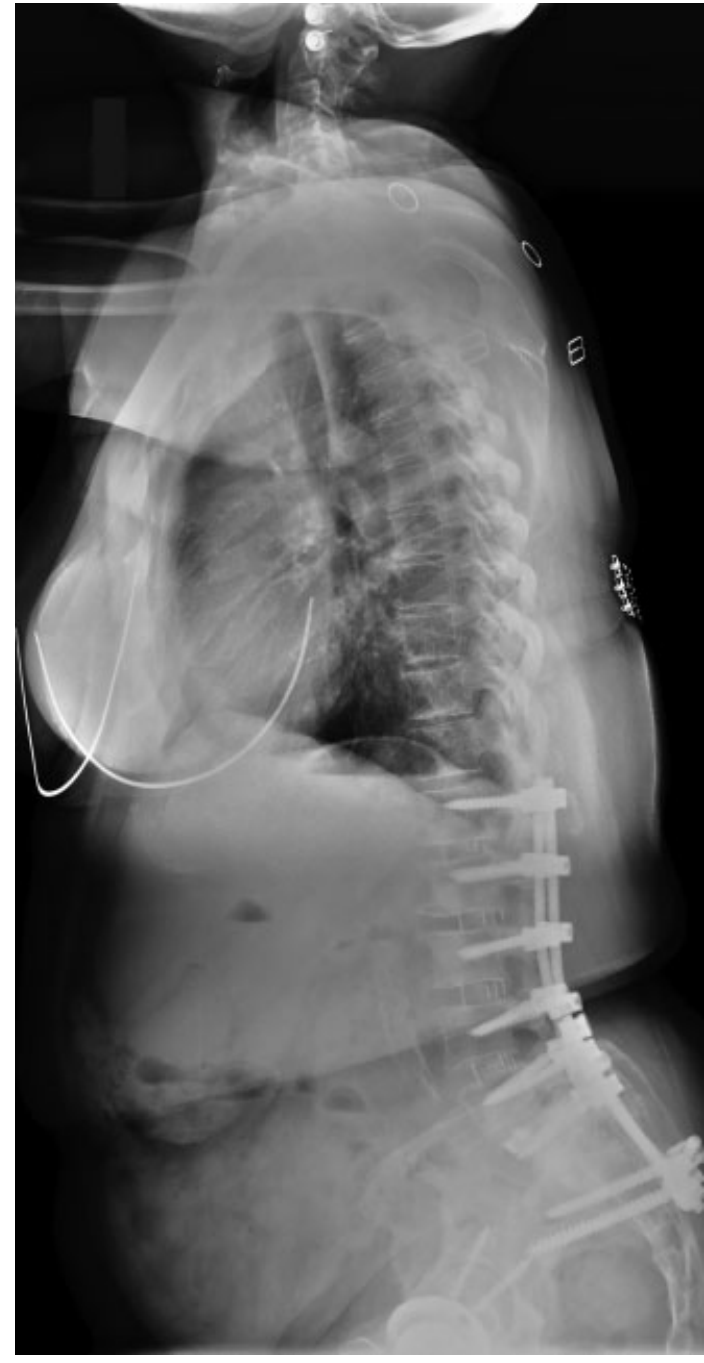

Fig. 13 A 6-month lateral X-ray image.

protein (rhBMP)-2 with the formation of a postoperative sterile seroma. ${ }^{3,8}$ It has been hypothesized that rhBMP-2 correlates with a higher rate of seroma formation due to the propensity for rhBMP-2 to cause inflammation. Repeat usage of rhBMP-2 has also been associated with seroma formation, which suggested a relationship to antibody formation. $^{3}$ This patient, however, received no rhBMP-2 to facilitate fusion. With few confounding variables to account for ( - Table $\mathbf{1}$ ), the causation of the recurring sterile seroma is still irresolute.

One possible factor to consider is an acute allergic reaction to the prophylactic vancomycin powder. Intravenous application of vancomycin has had a history of causing hypersensitivity reactions including red man syndrome and anaphylaxis. ${ }^{9}$ Studies have also described that a percentage of the population may be prone to releasing a large amount of histamine in response of vancomycin. ${ }^{9}$ Intravenous vancomycin has been shown to degranulate cutaneous mast cells and cause an area of flare in patients, ${ }^{9}$ which leads to questions of whether or not the topical vancomycin can cause an inflammatory reaction as well.
Visceral reactions that have been linked to the intravenous application of vancomycin include pseudarthrosis as well as occasional systemic reactions like nephrotoxicity. ${ }^{4}$ It was reported that the likelihood of systemic adverse effects is limited from $2 \mathrm{~g}$ of vancomycin since very low serum levels of the antibiotics have been measured in patients. ${ }^{4}$ Pseudarthrosis is a possible complication of the prophylactic powder since high concentrations of antibiotics are cytotoxic to cells; however, studies have shown no significant increase in the rate of pseudarthrosis in patients receiving vancomycin powder. ${ }^{3-5}$ Moreover, there is a lack of peer-reviewed studies addressing the adequate dosage of vancomycin powder to be placed within the wound leading to a discrepancy in determining appropriate dosage of the prophylactic powder. With the limitation of this study being a case study with sample size of one and lack of previous studies on this topic, analysis of a larger population on sterile seroma/hematoma formation with correlation to prophylactic vancomycin powder is warranted.

\section{Disclosures}

Jim A. Youssef, Board membership: Durango Orthopaedics; Consultancy: Nuvasive, Integra, Amedica; Royalties:

Nuvasive, Osprey Biomedical, Amedica, Integra

Douglas G. Orndorff, Consultancy: Nuvasive, Integra, Stryker, Amedica; Royalties: Integra

Morgan A. Scott, none

Rachel E. Ebner, none

Allison P. Knewitz, none

\section{Note}

The drug status is investigational because the drug in question in this article is not FDA approved for this indication.

This case report is exempt from IRB approval.

\section{Funding}

No funds were received in support of this work.

\section{References}

1 Hoffmann MF, Jones CB, Sietsema DL. Complications of rhBMP-2 utilization for posterolateral lumbar fusions requiring reoperation: a single practice, retrospective case series report. Spine J 2013;13(10):1244-1252

2 Martin JR, Adogwa O, Brown CR, et al. Experience with intrawound vancomycin powder for spinal deformity surgery. Spine (Phila Pa 1976) 2014;39(2):177-184

3 Tubaki VR, Rajasekaran S, Shetty AP. Effects of using intravenous antibiotic only versus local intrawound vancomycin antibiotic powder application in addition to intravenous antibiotics on postoperative infection in spine surgery in 907 patients. Spine (Phila Pa 1976) 2013;38(25):2149-2155

4 Strom RG, Pacione D, Kalhorn SP, Frempong-Boadu AK. Decreased risk of wound infection after posterior cervical fusion with routine local application of vancomycin powder. Spine (Phila Pa 1976) 2013;20(38):991-994 
5 Sweet FA, Roh M, Sliva C. Intrawound application of vancomycin for prophylaxis in instrumented thoracolumbar fusions: efficacy, drug levels, and patient outcomes. Spine (Phila Pa 1976) 2011; 36(24):2084-2088

6 Awad JN, Kebaish KM, Donigan J, Cohen DB, Kostuik JP. Analysis of the risk factors for the development of post-operative spinal epidural haematoma. J Bone Joint Surg Br 2005;87(9):1248-1252

7 Kanayama M, Oha F, Togawa D, Shigenobu K, Hashimoto T. Is closed-suction drainage necessary for single-level lumbar decompression?: review of 560 cases Clin Orthop Relat Res 2010;468(10):2690-2694

8 Hurlbert RJ, Alexander D, Bailey S, et al. rhBMP-2 for posterolateral instrumented lumbar fusion: a multicenter prospective randomized controlled trial. Spine (Phila Pa 1976) 2013; 38(25):2139-2148

9 An SY, Hwang EK, Kim JH, et al. Vancomycin-associated spontaneous cutaneous adverse drug reactions. Allergy Asthma Immunol Res 2011;3(3):194-198

\section{Editorial Perspective}

EBSJ welcomes this perplexing case report by Dr. Youssef and colleagues and the ensuing balanced commentary by Drs. Parker and Devin to foster further discussion on intraoperative topical vancomycin powder administration to reduce surgical site infections in spine care. The current evidence level does favor its administration in more invasive posterior surgeries, however, with some degree of uncertainty remaining. One useful application for case reports can lie in serving as an early warning report system for complications when more novel surgical technologies are being used, especially if complications are unusual in nature. In this case, as presented, EBSJ agrees with the commentators that it is premature to conclude that this patient suffered her recurrent seroma formation as a result of topical vancomycin powder formation. However, by presenting this case, hopefully EBSJ readers will place greater scrutiny on the indications and results of patients who have received topical vancomycin powder. 